\title{
Investigation of Energy Deposition and Dose Distributions on Collimator under Photon Beam
}

\author{
N. Demir ${ }^{a, *}, Z$ Z.N. KuluÖZTÜRK ${ }^{b}$ AND İ. AKKURT ${ }^{c}$ \\ ${ }^{a}$ Uludağ University, Department of Physics, Bursa, Turkey \\ ${ }^{b}$ Bitlis Eren University, Medical Imaging Techniques, Bitlis, Turkey \\ ${ }^{c}$ Süleyman Demirel University, Isparta, Turkey
}

\begin{abstract}
The bremsstrahlung photon facility where photon beam can be created via bremsstrahlung processes has been installed in many different accelerator in the world. TARLA (Turkish Accelerator and Radiation Laboratory at Ankara) will be first facility of TAC (Turkish Accelerator Center) project in Turkey. An optimal design of collimator is important for obtain a clean high energy photon beam in a bremsstrahlung facility. In this study the photon spectrum has been obtained depending on the entrance angle of the collimator. The energy deposition and dose distributions on the collimator by photon beam generated from $30 \mathrm{MeV}$ energy electron beam have been calculated with FLUKA Monte Carlo simulation code.
\end{abstract}

DOI: 10.12693/APhysPolA.128.B-400

PACS: 29.27.-a, 29.20.Ej

\section{Introduction}

The real photons produced at the electron linear accelerators are important tools for nuclear structures and variety nuclear astrophysics and medical physics experiments. Particularly, the photon scattering experiments are very useful for elementary excitations. Darmstadt electron accelerator S-DALINAC [1], linear accelerator facility in Gent university [2], superconducting electron accelerator ELBE [3] are some of the accelerator facilities working at photon-scattering experiments. The Turkish Accelerator and Radiation Laboratory (TARLA) in our country, is currently under construction, and also will be planned photon-scattering experiments in our bremsstrahlung facility [4]. In order to sensitive measurements, the photon beam is transported to the experimental area by a massive collimator system. It should be designed to minimize the spatial

distribution of photons and also to reduce the neutron production via $(\gamma, \mathrm{n})$ reactions at the collimator. It is important to measure energy deposited on the collimator in respect to safety.

In this work, the FLUKA simulation results are presented for collimator design in the TARLA bremsstrahlung facility. The photon spectrum has been investigated depending on the entrance radius. The energy deposition and dose distributions on the collimator by photon beam from $30 \mathrm{MeV}$ energy electron beam have been calculated.

\footnotetext{
* corresponding author; e-mail:

nlgndemir@gmail.com
}

\section{Materials and methods}

\subsection{Collimator}

We calculated the interactions of bremsstrahlung photons scattered from $6 \mu \mathrm{m}$ thick Ta radiator with collimator. This value is optimum thickness for radiator material [5]. In the simulation, we have choosen $\mathrm{Al}$ as the collimator material and it is positioned $1 \mathrm{~m}$ behind the radiator. The collimator has a length of $3200 \mathrm{~mm}$ and a cylindrical geometry with $0.25 \mathrm{~cm}$ inner, $5 \mathrm{~cm}$ outer radius.

\subsection{Simulation with FLUKA}

In the collimator system design is involved the electrons, bremsstrahlung photons interactions with various target materials. Also the photonuclear reactions in the collimator should be included depending on photon energy and collimator material. Effectively these complex processes can be calculated using the Monte Carlo code. FLUKA is the best choice for calculating these processes. It is a general purpose Monte Carlo code for particle transport and interactions and it can simulate interaction and diffusion in matter of about 60 different particle It used in many fields of physics for fundamental research and applications [6].

Energy deposition is expressed in $\mathrm{GeV} \mathrm{cm}^{-3}$ per unit primary weight by FLUKA. Radiation dose in the system thanks to energy deposition values were calculated by FLUKA. Doses is expressed in $\mathrm{GeV} / \mathrm{g}$ per unit primary weight. If it is expressed as Gy (Gray), multiply $\mathrm{GeV} / \mathrm{g}$ by $1.602176462 \times 10^{-7}[6]$.

\subsection{Calculations and Simulations}

The inputs for FLUKA are given as, an electron beam energy $30 \mathrm{MeV}$, beam shape pencil, beam direction along the $\mathrm{z}$ axis. The Ta radiator were positioned at $(0,0,0)$ in 
FLUKA geometry and it's radius and thickness, respectively, was choosen as $0.8 \mathrm{~cm}, 6 \mu \mathrm{m}$. Simulation were started by $5 \times 10^{6}$ primary electrons. Figure 1 represents the FLUKA geometry in the XZ plane and photon fluence in the whole geometry. The field covering Ta radiator and $\mathrm{Al}$ collimator was defined as the air.

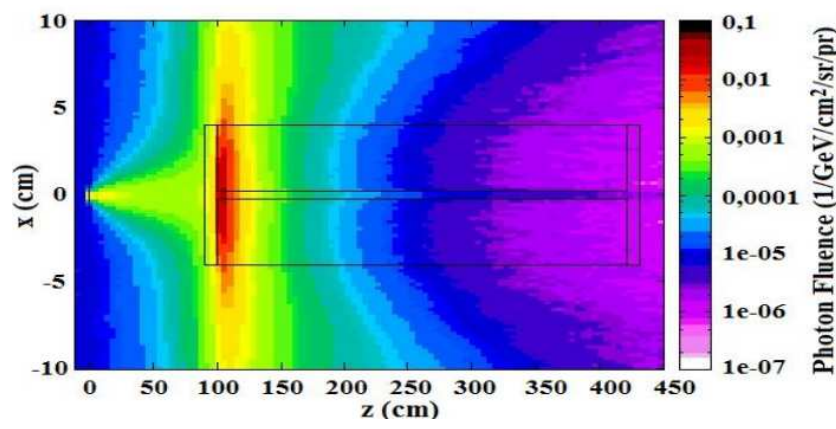

Fig. 1. The photon fluence in XZ plane.
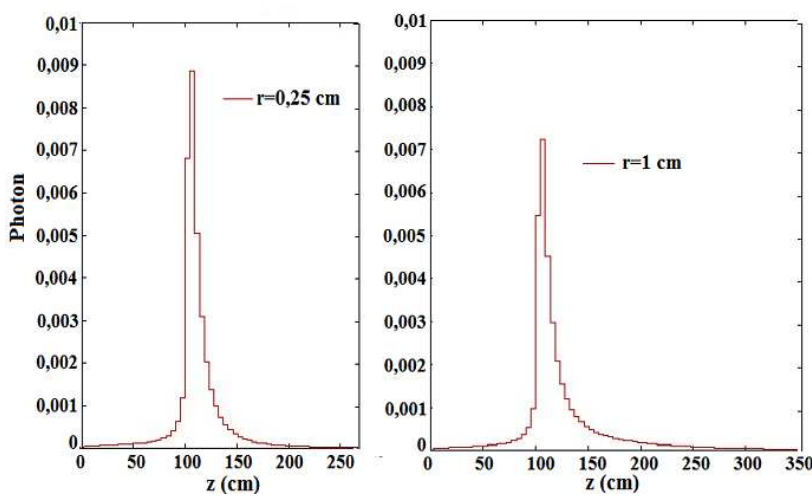

Fig. 2. The photon spectrum from the collimator for 0.25 and $1.0 \mathrm{~cm}$ entrance radii.

The photon spectrum was obtained for various entrance radii between 0.25 and $1.0 \mathrm{~cm}$. As shown in Fig. 2, the maximum photon was observed at the $0.25 \mathrm{~cm}$ radius. The tail of photon distribution is increased as the entrance radius is increased.

Deposited total energy and dose on the Al collimator was calculated for $0.25 \mathrm{~cm}$ entrance radius and plotted in Figs. 3 and 4 . The photon beam passing through the collimator leaves maximum energy, near the centre of the collimator. The maximum energy deposition is in the collimator on a radius of $1.8 \mathrm{~cm}$ and is due to secondaries. The maximum dose distribution is almost at the same radius.

\section{Conclusion}

Monte Carlo calculations were performed with FLUKA to optimize the collimator at the TARLA bremsstrahlung facility. Bremsstrahlung photons produced from electron beams with energy of $30 \mathrm{MeV}$ and the photon beam was

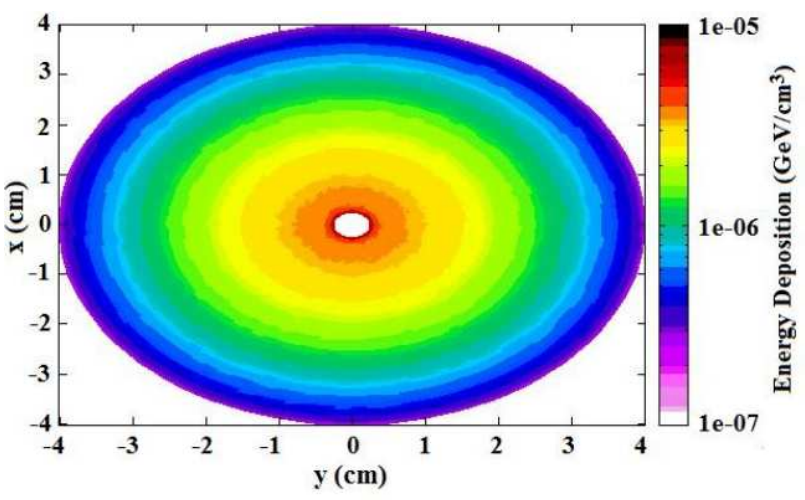

Fig. 3. The energy deposition profile in the $\mathrm{Al}$ collimator.

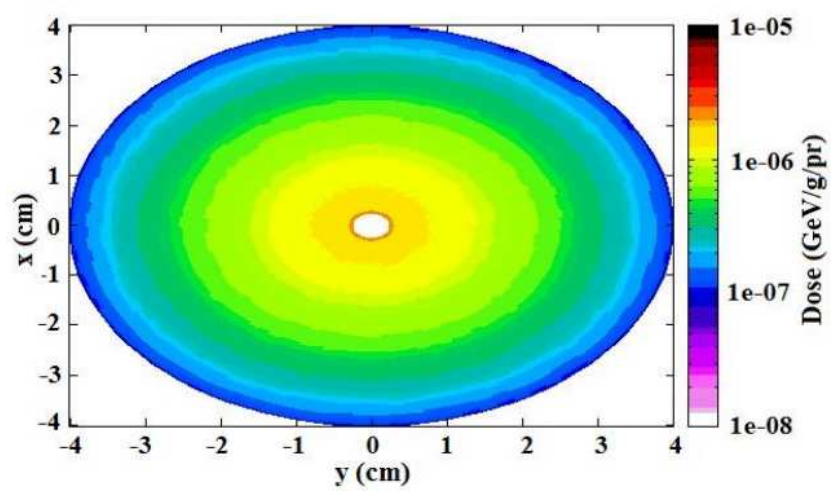

Fig. 4. The dose distribution profile in the $\mathrm{Al}$ collimator.

collimated by the Al collimator with a $3200 \mathrm{~mm}$ length and cylindrical geometry. It has been observed that the photon fluence increase and tail decrease with decreasing entrance radius. Thus, it is possible to obtain a cleaner high energy photon beam at the small radii. As a result maximum radiation dose and energy were formed the entrance of collimator and due to generation of secondary particle was observed to decreased toward outside of the collimator. In the next study, simulations will be developed for different collimator geometry and materials. Also, the $(\gamma, \mathrm{n})$ reactions will be included in the calculations and neutron fluence will be obtained.

\section{Acknowledgments}

This work has been supported by Suleyman Demirel University Foundation Unit (BAP) with the Project number 3407-D2-12.

\section{References}

[1] P. Mohr, J. Enders, T. Hartmann, H. Kaiser, D. Schiesser, S. Schmitt, S. Volz, F. Wissel, A. Zilges, Nucl. Instrum. Meth. A 423, 480 (1999). 
[2] W. Mondelaers, K. Van Laere, A. Goedefroot, K. Van den Bossche, Nucl. Instrum. Meth. A 368, 278 (1996).

[3] R. Schwengner, R. Beyera, F. Dönau, E. Grosse, A. Hartmann, A.R. Junghans, S. Mallion, G. Rusev, K.D. Schilling, W. Schulze, A. Wagner, Nucl. Instrum. Meth. A 555, 211 (2005).

[4] The Technical Design Report of Turkish Accerator and Radiation Laboratory at Ankara, TARLA - TDR 24.04.2011, Ankara 2011.
[5] N. Demir, Z.N. Demirci, I. Akkurt, Radiat. Eff. Defect. S. 168, 372 (2013).

[6] A. Ferrari, P.R. Sala, A. Fasso, J. Ranft, FLUKA: a multi-particle transport code, CERN 2005-10, Geneva 2005. 